\title{
Electromagnetic Actuator with Novel Electric Brake for Circuit Breaker
}

\author{
Byungjun Bae ${ }^{1}$ and Minjae Kim ${ }^{2 *}$ \\ ${ }^{1}$ Agency for Defense Development, Yuseong P.O. Box 35, Daejeon 34186, Republic of Korea \\ ${ }^{2}$ Department of Mechanical Engineering, Myongji University, Cheoin-gu, Yongin-si, Gyeonggi-do 17058, Republic of Korea
}

(Received 16 March 2016, Received in final form 12 August 2016, Accepted 18 August 2016)

\begin{abstract}
At the stroke end of an electromagnetic circuit breaker, the high speed of the mover makes a huge impact at the contact point, which induces the rebound problem of the mover that causes a breaker failure. Thus, a speed reduction equipment is required to address such problems. This study suggests to use an electric brake reduces the speed at the end of the stroke. The proposed circuit breaker which adopts the electric brake has a variable speed reduction function such that the continued rebound phenomenon ceases to occur. The electric brake is designed by the Finite Element Method (FEM) and the circuit and motion equations are solved using Time Difference Method (TDM). The comparisons between the simulation and experiments demonstrated the usefulness and validity of this study.
\end{abstract}

Keywords : circuit breaker, electromagnetic actuator, electric brake, dynamic characteristic analysis

\section{Introduction}

A circuit breaker serves the purpose of current breaking in an electric power system in order to protect the electric power system and electric machineries during the short circuit faults. It consists of an interrupter and mechanism parts as in Fig. 1, where the interrupter part removes the arc when the moving point of the contact is separated from the fixed point of the contact, and the mechanism part produces a tractive force during the contact point separation.

The conventional mechanical actuator and Permanent Magnetic Actuator (PMA) had some limitations and they could be addressed through a new type of Electro Magnetic Force driving Actuator [1]. This type of actuator facilitates lengthening of the stroke length in the mechanism part, thus making it available a high power actuator with small volume which would not have been feasible with existing actuators; therefore, it can be applied to a low voltage to a high voltage system $[2,3]$.

Generally, a circuit breaker with a long stroke has a very short duration of contact point opening. When we adopt the electromagnetic actuator in the circuit breaker that requires a long stroke, the circuit opening exerts the

(C)The Korean Magnetics Society. All rights reserved.

*Corresponding author: Tel: +82-31-324-1426

Fax: +82-31-330-6957, e-mail: minjk@mju.ac.kr electromagnetic force pushing the mover downwards so that the mover speed increases sharply at the end of the stroke; this phenomenon causes a huge mechanical impact on the stroke. This impact induces a rebound of the mover at the bottom of the stroke and the contact point experiences the failure of circuit breaking [4]. The rebound problem caused by no brake in the circuit breaker is similar to chattering in the switch or relay. And the continued rebound in the circuit breaker would bring about the circuit breaking failure (re-opening), high temperature by the collision, arc strike, and lifespan decrease of the system; in addition, an explosion can happen in the end $[5,6]$.

To remove this disadvantage, the spring add-on or mechanical oil damper could be adopted [7]; this, however, would imply the use of a mechanical actuator and thus cannot be considered a fundamental solution to the problem. Furthermore, the mechanical damage and limitations prohibit a mechanical actuator to be used as a damper.

This study proposes a novel electromagnetic actuator in the mechanism part to solve the high speed problem of the mover at the end of the stroke. The electric brake for the circuit breaker in this study adopts an additional brake coil that produces an Electric Motive Force (EMF) by means of the mover speed, and the external voltage by the capacitor aids stopping the mover at the end of the stroke [8]; accordingly, the failure of circuit breaking by the 
rebounds can be avoided. The proposed electric brake performance for the electromagnetic actuator is verified by the simulation and experiment such that the validity of the proposed system could be demonstrated.

The contributions of this study are as follows. 1) The suggested electric brake enables resolving the rebound problem in the electromagnetic circuit breaker which is favorable in the maintenance and reliability. 2) The induced EMF and a capacitor are used at once to produce a stable braking force such that a large braking force is now feasible. 3) Adjusting the brake current enables the brake to be used in the various kinds of braking speed profiles.

This paper is organized as follows. In Section 2, the electromagnetic actuator is introduced. Section 3 introduces the electric brake to be adopted. Section 4 discuss the simulation and experiment results of the electric brake. Finally, Section 5 provides concluding remarks.

\section{Electromagnetic Actuator for the Circuit Breaker}

This section describes the principle and construction of the electromagnetic actuator for the circuit breaker.

\subsection{Outline of the actuator}

Existing spring or oil pressure actuators have a low cost but poor reliability, and the large numbers of components lead to difficulty in their maintenance [9]. In the late 1990s, with the development of a permanent magnet type actuator (PMA) that had a simple structure with easy maintenance, the demerits of mechanical actuators were addressed, and the PMA could thus be used for the circuit breaker of a low voltage system [10]. PMA utilizes the electromagnetic force in the coil to induce a motion in the mover and the permanent magnets exert the holding force. However, the stroke constitutes the path of the magnetic flux and its length cannot exceed a certain limit for a given volume [11]. Therefore, PMA is not suitable for high voltage systems requiring long strokes.

To overcome such limitations of the PMA, the electromagnetic actuator using Lorentz force is suggested. In comparison with PMA, the electromagnetic actuator has the advantage of having a long stroke that expands the scope of application of such actuators from small voltage to ultra-high voltage applications. In the electromagnetic actuator, the air gap magnetic flux density does not change with the stroke variations and this is because the main magnet flux direction is perpendicular to the mover's direction of motion [2].

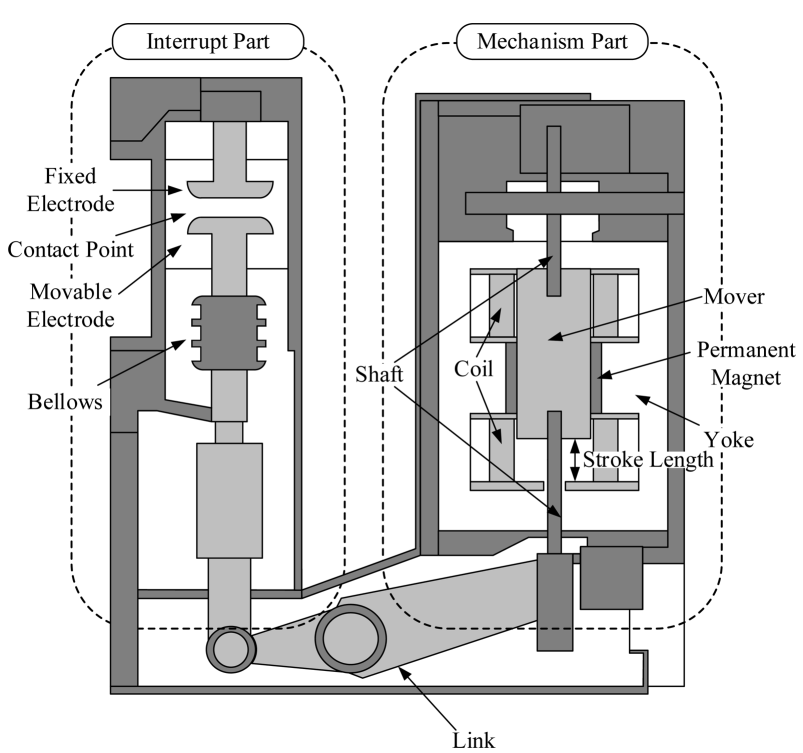

Fig. 1. Circuit breaker with interrupter and mechanism part.

\subsection{Principle and construction of the electromagnetic actuator}

Figure 2 shows the structure of the actuator mechanism system which replace the PMA mechanism part in Fig. 1. The stator core is layered, and contains dual (left and right) rectangular grooves inside which the mover is placed. The long magnets constitute the main permanent magnet that produces a flux perpendicular to the coil. The short magnets are the sub-permanent magnets that contribute towards the holding force required to fix the mover's movement [12]. The mover body comprises the coils sandwiched between cores above and below, held together by a urethane bonding.

The operating principle of the electromagnetic actuator is as follows: When the mover in Fig. 2 is on the top, the sub-permanent magnet maintains the mover's position

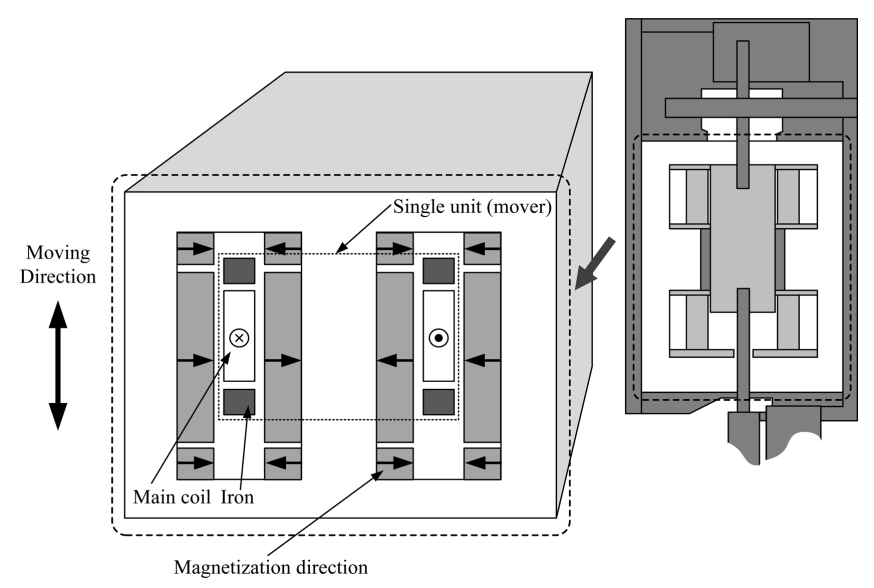

Fig. 2. Structure of the electromagnetic actuator. 
through the holding force. In this position, the magnetic flux by the sub-permanent magnet goes through the core, passes through the upper side of the stator core, and returns to the sub-permanent magnet making a closed path. As a result, the electromagnetic force holds the mover at the top side. This principle is similarly applicable when the mover is at the bottom.

The tractive force, i.e. the Lorentz force, of the mover is exerted by the electromagnets; the direction of current is perpendicular to the screen and the flow of magnetic flux is horizontal such that the force exerted on the coil is downwards if the current direction is as shown in Fig. 2. In the absence of an external force, if the Lorentz force is larger than the holding force, the mover starts to move as follows:

$$
\boldsymbol{F}_{\text {hold }}<\boldsymbol{F}_{\text {Lorentz }}
$$

where $\boldsymbol{F}_{\text {hold }}$ and $\boldsymbol{F}_{\text {Lorentz }}$ are the holding force and Lorentz force, respectively.

\section{Electric Brake for the Electromagnetic Actuator}

This section describes the need of the electric brake, presents its structure with the operating principle, analyzes its role, and shows the experimental system and method.

\subsection{Necessity of the electric brake}

The circuit breaker suffers from a rebound problem at the end of the stroke due to the high speed of the mover [13]. Such a rebound phenomenon can induce a failure of the circuit breaking, and therefore addressing the rebound problem is of critical importance while designing the circuit breaker [4]. However, the vibrations of a mechanical system cannot be easily eliminated, and therefore, as a general rule, a circuit breaker is designed with some limitation on the rebound length. This rebound problem applies not only for the mechanical actuator but also for the electromagnetic actuator. Accordingly, the mechanical damper was used for the electromagnetic actuator. However, using the mechanical damper in the electromagnetic actuator is equivalent to losing essential merits of such an actuator [7]. Mechanical brakes incur mechanical wear and damage proportional to the frequency of their use. Therefore, mechanical brakes require high maintenance and their reliability is uncertain. Consequently, a new actuator is required that utilizes the electric brake for reducing the stroke speed at the end of the stroke satisfying the speed limitation in the effective range. The electric brake operates using the non-contact braking caused by the Lorentz force where the problem of mechanical wear can be avoided. In addition, the electric brake is not affected by the stroke and therefore works appropriately wherever the mover operates.

\subsection{Principle and structure of the electric brake}

Figure 3 shows the concept structure of the proposed actuator. The proposed electric brake in this study is just below the main coil. The mark ' $A$ ' in Fig. 3 means the right side of mechanism part and is used again in Fig. 4 to identify the same side. Figure 4 shows 3-D shape of the system. When the braking is needed, the main coil becomes open, and the brake coil current flows in a direction opposite to that of the main coil. The direction of main coil current is not reversed for the braking because the large inductance of the main coil opposes a change in the direction of instantaneous current flow; thus, additional coil is used for the brake which has less inductance, resistance, and turns [14].

Figure 5 shows the equivalent circuit of the actuator with the electric brake. The circuit breaker operation starts on the occurrence of an accidental change in the

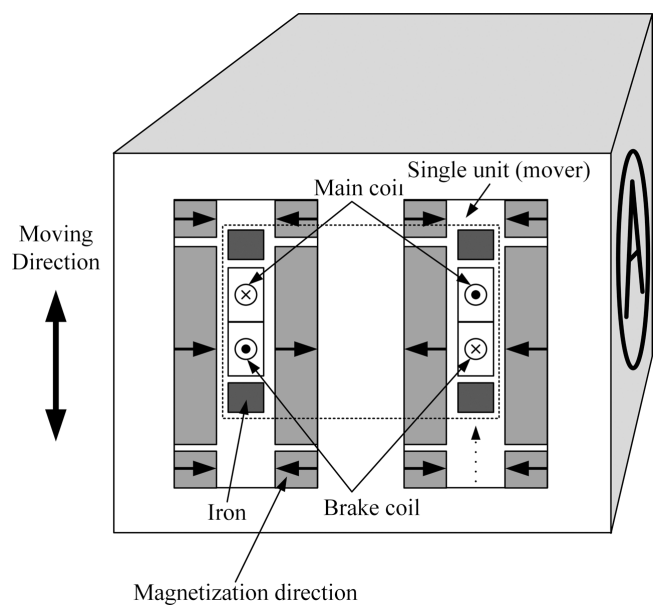

Fig. 3. Structure of the proposed electric brake.

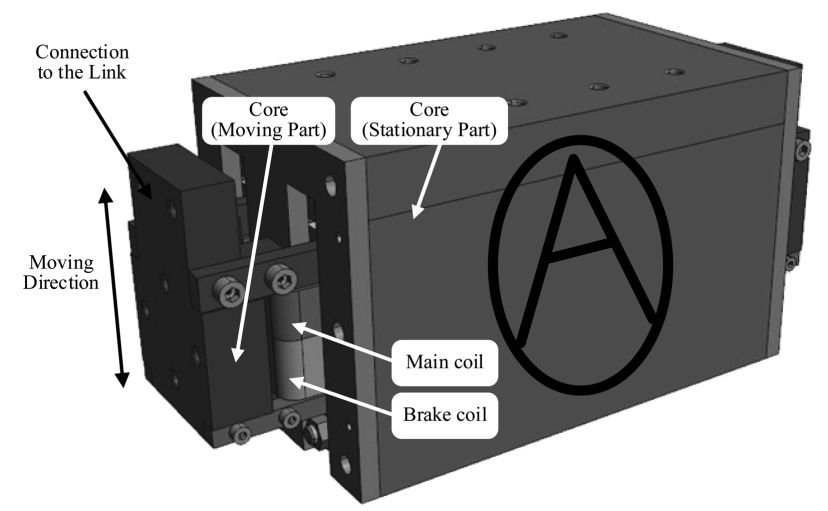

Fig. 4. 3-D shape of the electromagnetic actuator with the electric brake. 


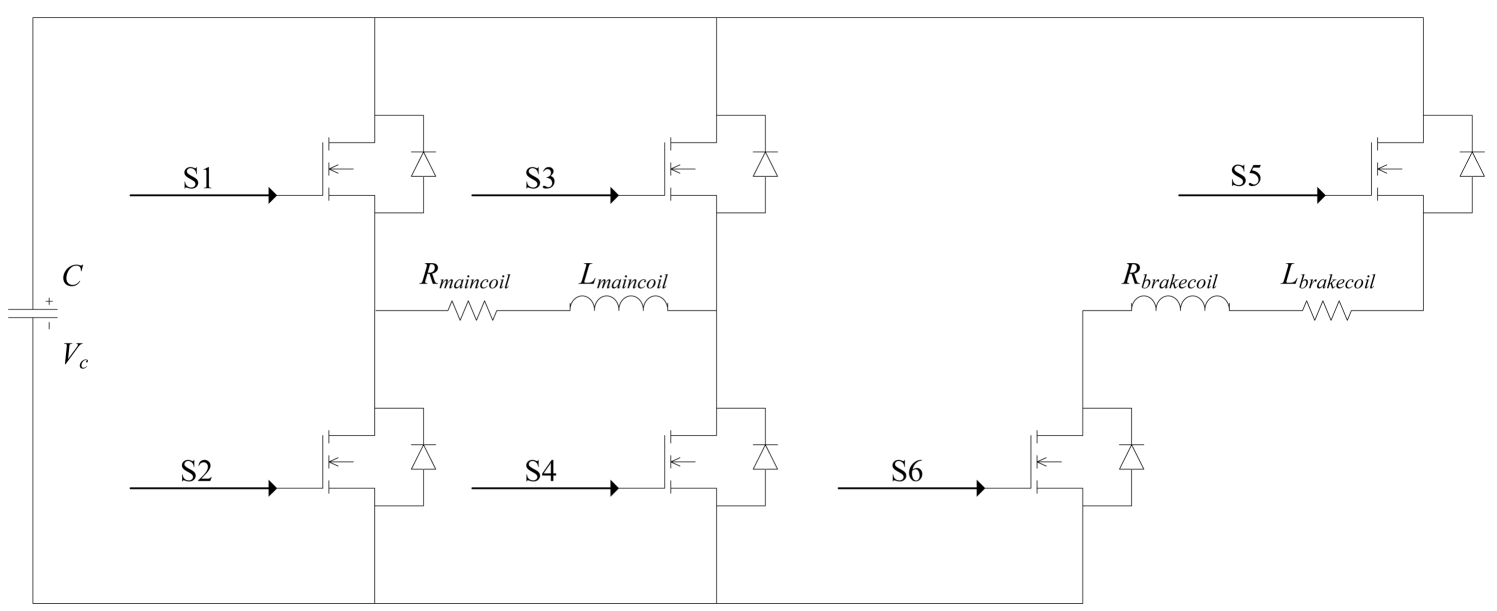

Fig. 5. Equivalent circuit of the actuator with the electric brake.

electric system and the induced EMF and capacitor are used as the energy source to produce the braking force. Consequently, producing a very large braking force is feasible. The switches S1 and S4 serve for circuit opening, while S2 and S3 serve for circuit closing. When the brake is on, $\mathrm{S} 1$ and S4 are off and S5 and S6 are on, where the on-times for current flow in the main coil and brake coils can be adjusted to satisfy the required speed.

\subsection{Numerical analysis}

To analyze the operating character of the electromagnetic actuator, static magnetic field analysis and solution of the circuit and motion equations are required. This study uses the 2-D Finite Element Method (FEM) such that the static magnetic field of the permanent magnet and the external current source are analyzed, followed by solution of the circuit and motion equations using TDM (Time Difference Method). To solve the circuit and motion equations, the flux linkage and force on the mover are required. These physical quantities are resolved through post-processing after the static magnetic field analysis as given in Fig. 6 . The electromagnetic force of the mover and the Lorentz force of the coil are solved by the Maxwell stress tensor method. Then, the circuit and motion equations are solved using TDM; the current in the coil and the voltage in the capacitor are calculated by the circuit equation, and the mover's displacement and velocity are calculated by the motion equation.

1) Static magnetic field analysis by FEM

The governing equation of the magnetic static field for the external current source and permanent magnet is as
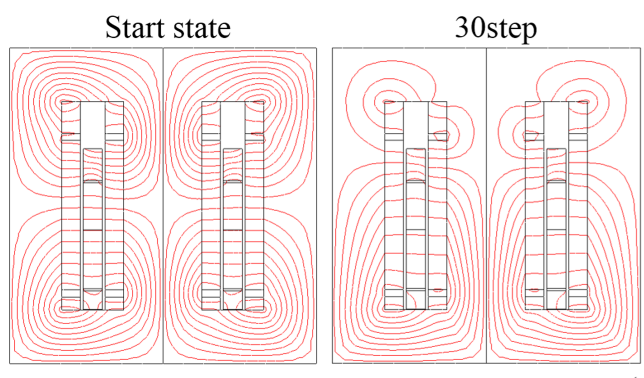

(a)

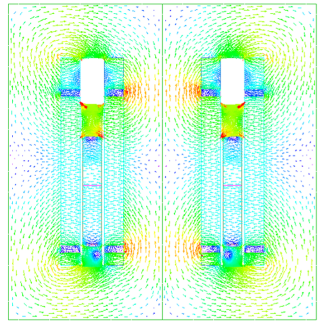

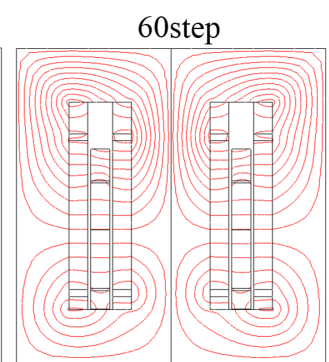

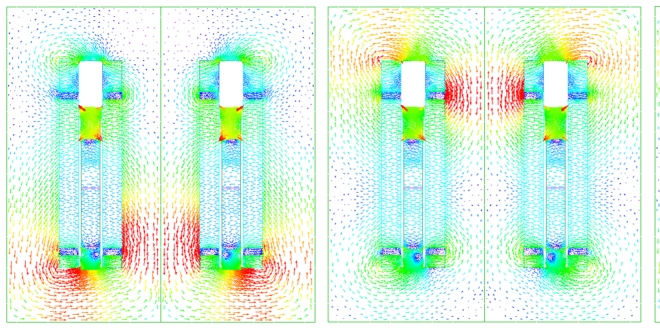

(b)
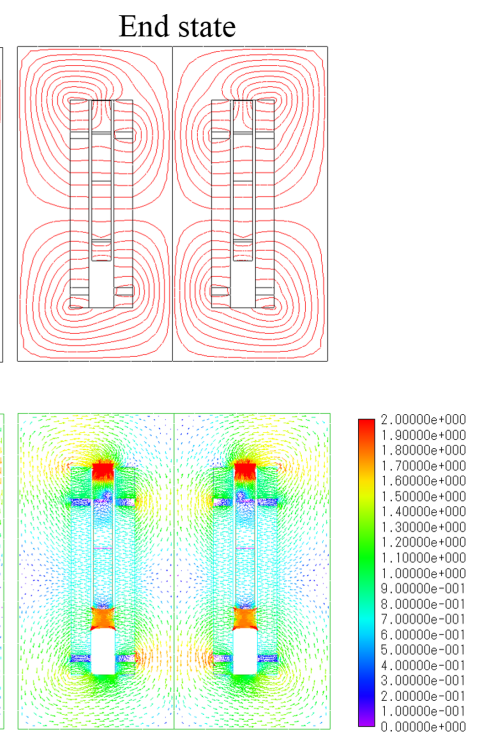

Fig. 6. (Color online) Numerical Analysis of Circuit breaker (a) Lines of magnetic force and (b) Magnetic flux density. 
follows:

$$
\nabla \times v(\nabla \times \boldsymbol{A})=\boldsymbol{J}_{0}+\nabla \times\left(v \mu_{0} \boldsymbol{M}\right)
$$

where, $v, \boldsymbol{A}, \boldsymbol{J}_{0}, \boldsymbol{M}$, and $\mu_{0}$ are the reluctivity as the reciprocal of permeability, magnetic vector potential, magnetization, and permeability of the free space, respectively. The first and second terms on the right-hand side denote the current densities of the external current source and the permanent magnet [15-17].

The magnetic vector potential $\boldsymbol{A}$ acquired by solving the governing equation using FEM in the static magnetic field is used for calculating the flux linkage and Lorentz force. The flux linkage can be written as (3) which integrates the magnetic potential using a curvilinear integral.

$$
\Phi=\int_{S}(\nabla \times \boldsymbol{A}) \cdot d \boldsymbol{S}=\oint \boldsymbol{A} \cdot d \boldsymbol{L}
$$

where, $\Phi, \boldsymbol{S}$, and $\boldsymbol{L}$ are the magnetic flux, area for the flux linkage, and length of coil winding path, respectively. The force to the mover is calculated by the Maxwell stress tensor method as follows [18]:

$$
\boldsymbol{F}=\iint_{S}\left(\frac{1}{\mu_{0}}(\boldsymbol{B} \cdot \boldsymbol{n}) \boldsymbol{B}-\frac{1}{2 \mu_{0}}\left(B^{2} \boldsymbol{n}\right)\right) d S
$$

where $\boldsymbol{n}$, and $\boldsymbol{B}$ are the unit vector perpendicular to the closed surface curve around the mover, and magnetic flux density. The force obtained by the tensor method is used to solve the motion equation.

2) Solving the circuit and motion equations

To analyze the motion of the electromagnetic actuator, the equivalent circuit and motion equations should be calculated in advance. In the equivalent circuit, the induced EMF in the electromagnetic actuator is produced by both the current variation (transformer voltage) and the motion of the mover (speed voltage). The circuit equation of the main coil is defined as follows:

$$
V_{c}=i R_{\text {maincoil }}+L_{x, \text { maincoil }} \frac{d x}{d t}+L_{i, \text { maincoil }} \frac{d i}{d t}
$$

The second term is the induced EMF by the current variation, and the third term is the induced EMF by the speed variation. $V_{c}, R_{\text {maincoil }}, L_{i, \text { maincoil }}$, and $L_{x, \text { maincoil }}$ are the charged voltage at the capacitor, resistance of the main coil, inductance with respect to the current in the main coil, and inductance with respect to the displacement of the mover in the main coil, respectively. The variation of flux interlinkage is calculated by the static magnetic flux analysis.

The circuit equation for the brake coil is similar to the

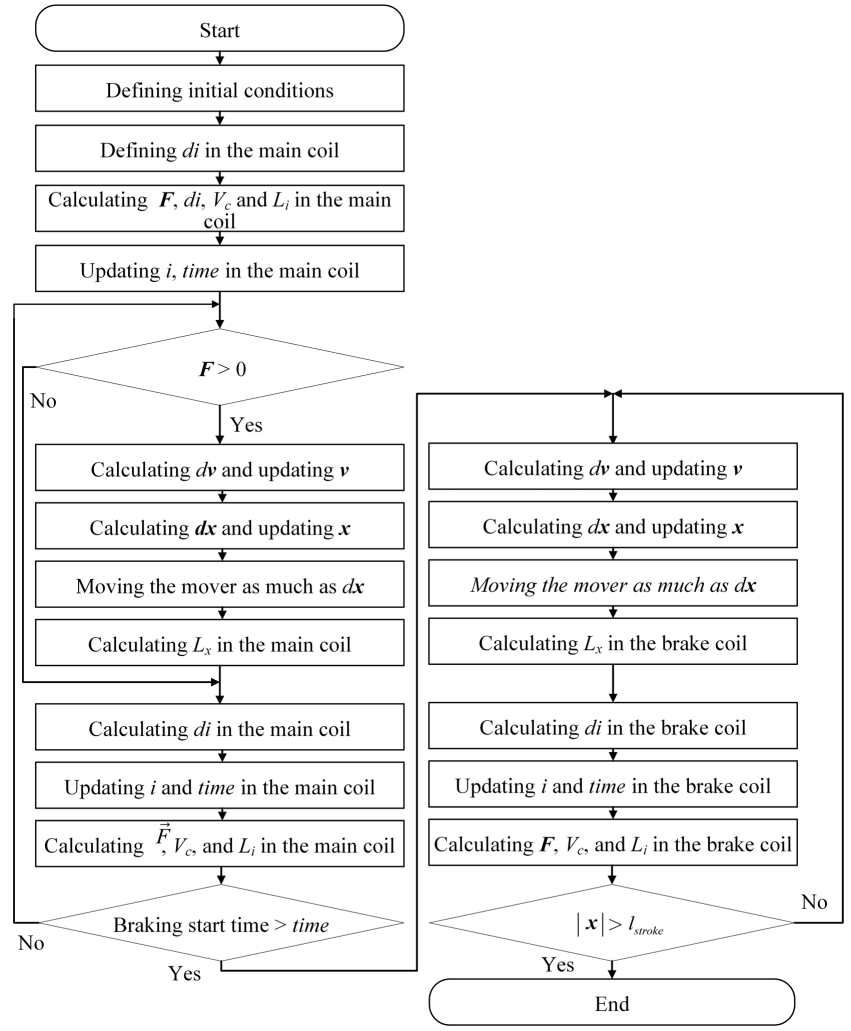

Fig. 7. Flowchart of dynamic characteristic analysis.

circuit equation of the main coil except for the term of speed voltage being added to the capacitor voltage as follows:

$$
V_{c}+L_{x, \text { brakecoil }} \frac{d x}{d t}=i R_{\text {brakecoil }}+L_{i, \text { brakecoil }} \frac{d i}{d t}
$$

where $R_{\text {brakecoil }}, L_{i, \text { brakecoil }}$, and $L_{x, \text { brakecoil }}$ are is resistance of the brake coil flux, linkage variations with respect to the current in the brake coil, and flux linkage variations with respect to the displacement of the mover in the brake coil, respectively. The speed and distance of the mover is calculated by the motion equation using (7), where it is assumed that the mover has a constant acceleration in one step.

$$
m(\boldsymbol{a}+\boldsymbol{g})=\boldsymbol{F}_{\text {Lorentz }}+\boldsymbol{F}_{\text {hold }}+\boldsymbol{F}_{\text {fric }}+\boldsymbol{F}_{\text {spring }}
$$

$m, \boldsymbol{a}, \boldsymbol{g}, \boldsymbol{F}_{\text {Lorentz }}, \boldsymbol{F}_{\text {hold }}, \boldsymbol{F}_{\text {fric }}, \boldsymbol{F}_{\text {spring }}$ are the mass of the mover, acceleration of the mover, gravity, Lorentz force on the coil, holding force on the mover coil, friction force, and contact force of the spring at the contact point, respectively. The speed or distance of the mover is then calculated using (7) and the motion equation could be solved step by step using TDM. The flowchart of dynamic characteristic analysis is given in Fig. 7. 
Table 1. Specification of the model.

\begin{tabular}{|c|c|c|}
\hline \multicolumn{2}{|c|}{ Division } & Specification \\
\hline \multicolumn{2}{|c|}{ Closing holding force $(\mathrm{N})$} & 4000 \\
\hline \multicolumn{2}{|c|}{ Opening holding force $(\mathrm{N})$} & 800 \\
\hline \multicolumn{2}{|c|}{ Stroke $(\mathrm{mm})$} & 17.4 \\
\hline \multicolumn{2}{|c|}{ Capacitance (F) } & $0.1 \times 2$ \\
\hline \multicolumn{2}{|c|}{ Operating capacitor voltage (V) } & 100 \\
\hline \multirow{3}{*}{ Size } & Width (mm) & 130.4 \\
\hline & Height (mm) & 134.7 \\
\hline & Depth (mm) & 200 \\
\hline \multirow{3}{*}{ Main coil } & Diameter (mm) & 1 \\
\hline & Resistance $(\Omega)$ & 1.88 \\
\hline & Turn count & 144 \\
\hline \multirow{3}{*}{ Brake coil } & Diameter (mm) & 1.5 \\
\hline & Resistance $(\Omega)$ & 0.44 \\
\hline & Turn count & 75 \\
\hline
\end{tabular}

\subsection{Experimental System and Method}

The experiment focuses on the verification of the brake performance with the mechanism part, where the detailed design specifications of the actuator are given in Table 1. Figure 8 shows the experimental system, and (a) and (b) mean the front and side view of the mechanism part. This mechanism part would be applied to various kinds of interrupt parts from the low voltage to the high voltage afterwards. To guarantee a linear movement, a guide is set up inside and the braking effect was evaluated by gradual increase of braking time (duration of the braking current flow); the design object is to meet the minimum average speed of $1.5 \mathrm{~m} / \mathrm{s}$.

The distance of actuator was measured by the electric actuator distance measurement equipment of MEL laser sensor M7 type as in Fig. 8(b). The degree of precision is

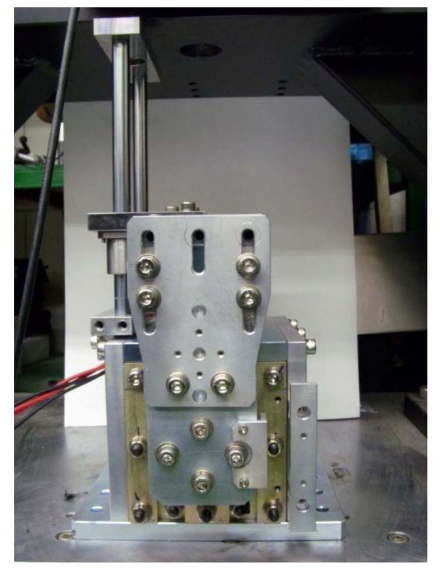

(a)

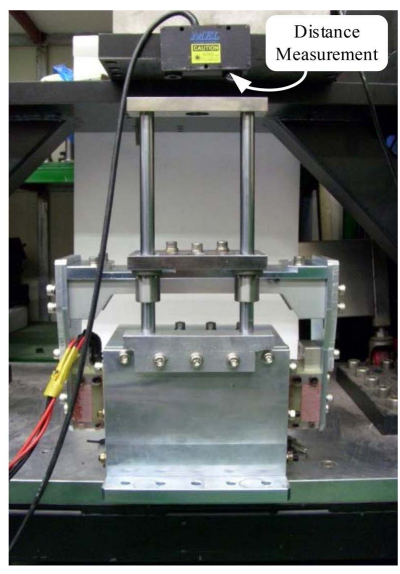

(b)
Fig. 8. (Color online) Electromagnetic actuator (a) Front view and (b) Side view.

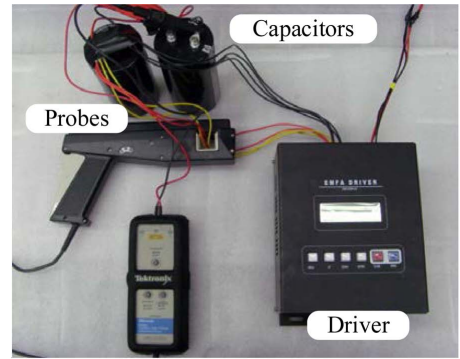

(a)

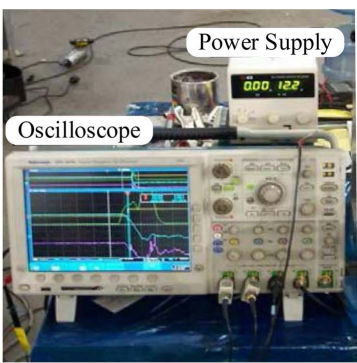

(b)
Fig. 9. (Color online) Experimental equipment (a) Capacitor, probes, and driver and (b) Oscilloscope and power supply.

$0.0002 \mathrm{~mm}$, and a response frequency is $10 \mathrm{kHz}$. Figure 9(a) shows the driver, capacitor, and noncontact probes for the current measurement and the voltage, current, and displacement were identified by the oscilloscope in Fig. 9(b).

\section{Results and Discussions}

Figure 10 displays a comparison between experimental and simulation results of the stroke when the electric brake is not applied. In the experiment results, the mover stroke finishes its operation at $36 \mathrm{~ms}$ followed by the rebound and the rebound size is $1.5 \mathrm{~mm}$ (gap between the top and bottom). The simulation analysis results display a pattern very similar to the experiment results, and the operation time is $35 \mathrm{~ms}$ with an error about $1 \mathrm{~ms}$ as compared to the experiment results. Because the simulation analysis was done using FEM, the impact bound analysis was not feasible and the mover reaches the stable state immediately after completion of its operation.

Figure 11 to 13 display a comparison between experimental and simulation results when the electric brake is applied during $5 \mathrm{~ms}$. Figure 11 shows the current of the main coil. The current is fed during $28 \mathrm{~ms}$ and the maximum current is $30 \mathrm{~A}$ in the experiments and $35 \mathrm{~A}$ in

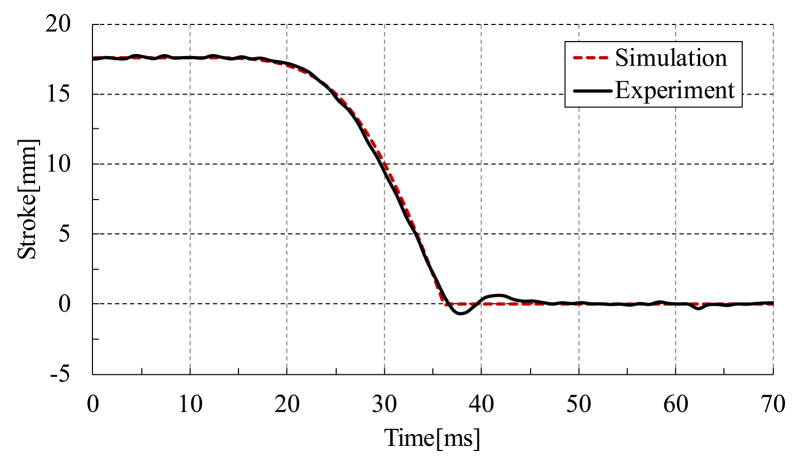

Fig. 10. (Color online) Comparison of stroke during opening operation without brake. 


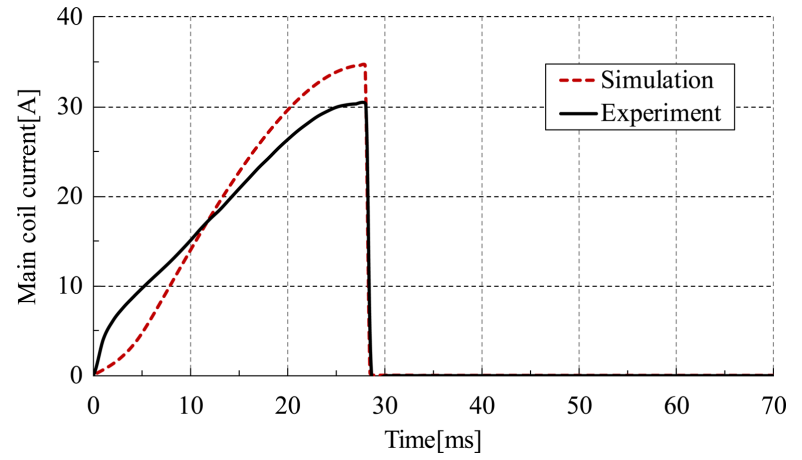

Fig. 11. (Color online) Comparison of main coil current during opening operation.

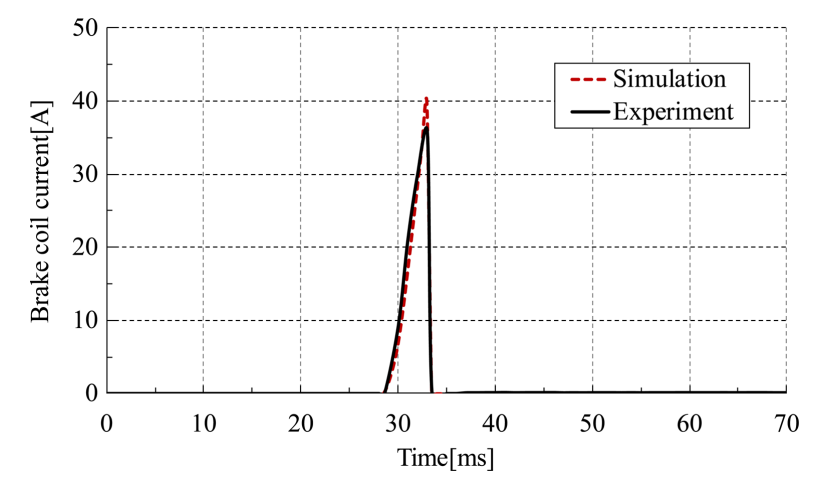

Fig. 12. (Color online) Comparison of brake coil current during opening operation.

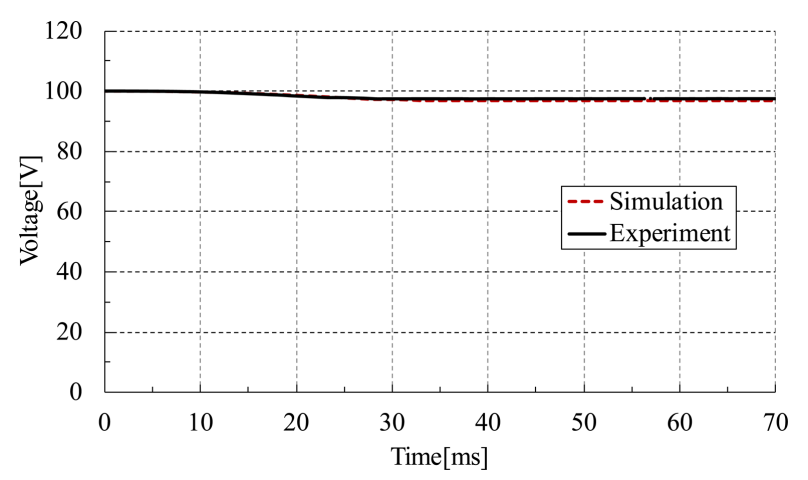

Fig. 13. (Color online) Comparison of voltage during opening operation.

the simulation, thus exhibiting a difference. This is because the thread groove for the layered core disturbs the flux flow and stroke of the mover. The current before the mover motion increases rapidly due to action of the EMF alone. However, the rate of increase in current decreases as the back EMF begins to work. Figure 12 shows a comparison for the current in the brake coil. After $28 \mathrm{~ms}$, the brake voltage is on, and the braking completes $5 \mathrm{~ms}$ later. To create an effective braking effect during the short time, a steep increase in current is essential. The brake

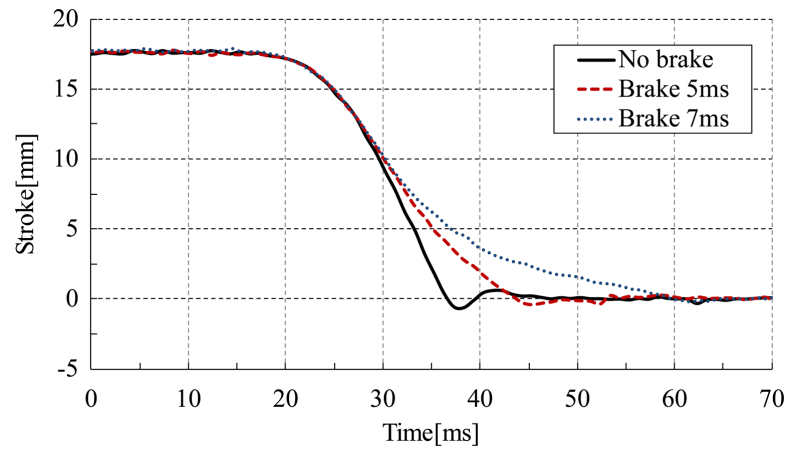

Fig. 14. (Color online) Experimental result on comparison of stroke according to braking time.

Table 2. Experimental results in terms of braking time.

\begin{tabular}{cccc}
\hline \hline Division & $\begin{array}{c}\text { Main coil current } \\
\text { on time }\end{array}$ & $\begin{array}{c}\text { Brake coil current } \\
\text { on time }\end{array}$ & $\begin{array}{c}\text { Finishing } \\
\text { time }\end{array}$ \\
\hline Without brake & $40 \mathrm{~ms}$ & $0 \mathrm{~ms}$ & $36 \mathrm{~ms}$ \\
\hline \multirow{2}{*}{ With brake } & $28 \mathrm{~ms}$ & $5 \mathrm{~ms}$ & $44 \mathrm{~ms}$ \\
& $28 \mathrm{~ms}$ & $7 \mathrm{~ms}$ & $60 \mathrm{~ms}$ \\
\hline
\end{tabular}

coil has a relatively small turn numbers in comparison with the main coil such that the rapid current increase is feasible. And the resultant gaps between the experiment and simulation does not show a huge error. Figure 13 shows the voltage of the capacitor. The capacitor voltage decreases as the current flow increases and hence the gaps from the initial condition $100 \mathrm{~V}$ is about $1.5 \mathrm{~V}$ in both simulation and experiment when the system is in the steady state.

Figure 14 shows the experimental braking performance when the brake duration is $5 \mathrm{~ms}$ including no braking and $7 \mathrm{~ms}$ braking. The duration of the main coil current was $40 \mathrm{~ms}$ when the brake is off to cease the first big rebound. And $28 \mathrm{~ms}$ was applied when the brake is on, where the speed reduction of the mover is clearly in accordance with the brake time. The finishing time is $55 \mathrm{~ms}$ when the brake duration is $5 \mathrm{~ms}$ and is $60 \mathrm{~ms}$ when the brake duration is $7 \mathrm{~ms}$. As the electric braking duration becomes longer, the rebound decreases further while the duration is still realistic [19-22]. Table 2 shows the experimental condition and finishing time of these operations. As a result, the electric brake could be used for the speed reduction at the end of the stroke and the performance of the proposed brake could be validated.

\section{Conclusion}

This study proposed a new type of electric brake for a circuit breaker with an electromagnetic actuator. The proposed brake overcomes the existing problem such that 
the effective reduction of the mover speed could be guaranteed. The analysis of operation characteristics was done using FEM and TDM. FEM was used for the static magnetic field analysis of the PM and current source; the induced Lorentz force, and inductance were subsequently used in the circuit and motion equations using TDM. The comparison between simulation and experimental results verify the validity and usefulness of the proposed electric brake. And the motion of the mover is much smoother when we use the proposed electric brake. This study aims at the identification of such smooth mechanism focusing on the mechanism part and the follow-up studies would evaluate the performance of this system for various circumstances from low voltage to high voltage in the future.

\section{References}

[1] J. H. Kang, D. K. Shin, H. K. Jung, K. H. Kim, W. Y. Lee, and J. S. Chun, International Symposium on Linear Drives for Industry Applications (LDIA) (2005) pp. 359362.

[2] J. H. Kang, Ph.D. Thesis, Seoul National University, Korea (2009).

[3] D. J. Cho, S. K. Hong, D. K. Woo, and H. K. Jung, International Conference on Electrical Machines and Systems (ICEMS) (2011) pp. 1-4.

[4] E. Dullni and S. F. Zhao, International Symposium on Discharges and Electrical Insulation in Vacuum (ISDEIV) (2010) pp. 463-466.

[5] W. Shujuan, R. Wanbin, and F. Weiwei, Electric Contacts (2004) pp. 453-458.

[6] L. Sun, Z. Wang, S. He, Y. Geng, and Z. Liu, Proc. International Conference on Electric Power EquipmentSwitching Technology (ICEPE-ST) (2014) pp. 1-4.
[7] B. T. Bae, S. H. Lee, J. E. Park, J. Y. Kim, and J. H. Kim, Proceeding of KSME (2012) pp. 2226-2229.

[8] J. S. Kim, S. M. Kim, J. H. Jeong, S. C. Jeong, and J. W. Lee, Int. J. Automot. Techn. 17, 865 (2016).

[9] R. E. Kim, M. S. Thesis, Seoul National University, Korea (2009).

[10] D. K. Shin, M.S. Thesis, Seoul National University, Korea (2006).

[11] C. H. Lee, B. H. Shin, and Y. B. Bang, IEEE Trans. Ind. Eng. 63, 1655 (2016).

[12] S. K. Hong, B. J. Bae, D. J. Cho, and H. K. Jung, International Conference on Electrical Machines and Systems (ICEMS) (2010) pp. 1573-1577.

[13] W. J. Park, K. Y. Ahn, and H. I. Yang, Proceeding of KSME (2010) pp. 956-961.

[14] H. C. Yi, K. I. Hwang, J. H. Kim, and J. H. Kim, J. Magn. 18, 466 (2013).

[15] D. H. Im, Finite element method in the electric field, Dong Myeong Publishers, Seoul (1987).

[16] S. J. Salon, Finite element analysis of electrical machines, Kluwer academic publishers, Boston (1995).

[17] A. J. Davies, The finite element method: a first approach, Oxford University Press, New York (1980).

[18] H. H. Woodson and J. Melcher, Electromechanical Dynamics Part II: Fields, Forces, and Motion, Wiley, New York (1986).

[19] E. Dong, T. Qin, Y. Wang, X. Duan, and J. Zou, IEEE Trans. Pow. Del. 63, 2594 (2013).

[20] Z. Wang, L. Sun, S. He, Y. Geng, and Z. Liu, IEEE Trans. Magn. 50, 129 (2014).

[21] L. Wei, F. Chun-en, Z. Lili, and W. Jun, Discharges and Electrical Insulation in Vacuum (ISDEIV) (2008) pp. $125-128$

[22] F. Liu, H. Guo, Q. Yang, L. Zhang, and W. Yan, IEEE Trans. Appl. Supercond. 14, 1918 (2004). 